Macià Bordalba, M. y Garreta Bochaca, J. (2018). Accesibilidad y alfabetización digital: barreras para la integración de las TIC en la comunicación familia/escuela. Revista de Investigación Educativa, 36(1), 239-257.

DOI: http://dx.doi.org/10.6018/rie.36.1.290111

\title{
Accesibilidad y alfabetización digital: barreras para la integración de las TIC en la comunicación familia/escuela
}

\section{Access and digital literacy: barriers of the integration of ICT in family/school communication}

\author{
Mònica Macià Bordalba y Jordi Garreta Bochaca \\ Departamento de Geografía y Sociología. Facultad de Educación, Psicología y Trabajo Social. Universidad de Lleida
}

(España)

\begin{abstract}
Resumen
Los recursos tecnológicos han ido apareciendo en los centros escolares como potenciales instrumentos para mejorar la información y la comunicación con las familias. Una comunicación que es esencial para mejorar la relación entre ambos agentes e incrementar la implicación y participación de los progenitores en la educación de sus hijos y en el centro. En este contexto, el presente trabajo tiene como objetivos identificar los factores que influyen negativamente en el uso comunicativo de las TIC y determinar si los estudios realizados en el campo pedagógico (de aplicación de las TIC en los procesos educativos de aula) pueden aplicarse en el ámbito comunicacional con las familias. Para ello se ha llevado a cabo un estudio etnográfico en 20 centros de educación primaria (realizado en cuatro Comunidades Autónomas españolas), seleccionados por tener indicadores positivos de implicación de las familias en los centros. Los resultados muestran que el acceso de las familias, así como la formación y actitudes de padres y docentes hacia las TIC, condicionan el uso de las nuevas tecnologías en la comunicación familia-escuela. La investigación evidencia, pues, que no solo

Correspondencia: Mònica Macià Bordalba, monica.macia@geosoc.udl.cat, Avenida de 1'Estudi General, 4, 25001, Lleida.

Artículo vinculado al Proyecto de Investigación Familias y Escuelas. Discursos y prácticas cotidianas sobre la participación en la educación obligatoria (referencia: EDU2012-32657). Se inscribe también dentro del Programa de Formación de Profesorado Universitario del Ministerio de Educación, Cultura y Deporte (referencia: FPU13/02456).
\end{abstract}


se debe mejorar la formación de las familias, sino también la de los docentes, especialmente para reducir su resistencia a utilizarlas (se requiere, sobre todo, una formación en actitudes).

Palabras clave: relación familia-escuela; comunicación mediada por ordenador; alfabetización digital; accesibilidad; barreras; educación primaria.

\section{Abstract}

Technological resources have gradually appeared in schools as potentially useful instruments to better communicate information to families. This communication is essential to improve the relationship between parents and teachers and to increase the involvement of families in their children's education and participation in schools. In this context, the article aims to identify the factors that negatively influence the communicative use of digital tools as well as to know whether the studies about the integration of ICT as pedagogical resources could be applied to the communicational field between families and schools. Based on an ethnographic study in 20 primary centres (located in Aragón, Cataluña, La Rioja y las Islas Baleares), which were selected for their positive indicators concerning the parental involvement, it was concluded that families' access as well as training and attitudes of parents and teaching staff influence the degree of ICT use as a means of communication. The research shows that the lack of training in ICT among families is important, but also teachers should be trained, especially to reduce resistance about using digital means of communication. Thus, it is recommended to stimulate accessibility and digital literacy among teachers and families to enhance the use of ICT in order to strengthen parent-school partnership.

Keywords: family-school relationship; computer mediated communication; computer literacy; accessibility; barriers; elementary schools.

\section{Introducción}

Vivimos en la sociedad de la información y del conocimiento (Burch, 2005). También en la sociedad líquida (Bauman, 2003) y en la sociedad red (Castells, 2006). Incluso hay quien la acuña sociedad de la ignorancia (Brey, Innerarity y Mayos, 2009). Diferentes términos que observan desde diferentes perspectivas la sociedad del siglo XXI, pero cuyos ojos convergen en un vector común: la demanda a sus miembros de una alfabetización informacional. Y tecnológica. Y digital. Y mediática. Y audiovisual. De nuevo, muchos términos impulsados desde múltiples ámbitos científicos y que ensalzan distintas necesidades competenciales (ver González, Gozálvez y Ramírez, 2014; Lee y So, 2014) pero construidos sobre un mismo pilar: la voluntad de crear ciudadanos que se formen a lo largo de toda la vida. Ciudadanos cultos y democráticos, capaces de socializarse en los nuevos sistemas comunicacionales y de desarrollarse en la nueva cultura digital.

Nuevas necesidades, nuevos retos y nuevas respuestas educativas. En España, el Programa Escuela 2.0 (2009-2012) ha sido la materialización de las políticas basadas en el modelo 1:1 o un ordenador por alumno, que tuvieron un amplio desarrollo a nivel internacional en la primera década del siglo XXI. Estas políticas pretendían la dotación masiva de recursos tecnológicos en las aulas y la formación del profesorado, con el objetivo último de conseguir la integración pedagógica de las Tecnologías de la Información y la Comunicación (TIC) en los procesos educativos (Area et al., 2014). Pero el 
legado que han dejado estos programas ha sido básicamente material (Tirado-Morueta y Aguaded-Gómez, 2014). Los cambios acontecidos en las metodologías educativas han quedado en un segundo plano y las herramientas digitales se siguen utilizando, sobre todo, como un elemento anexo y complementario a la docencia, sin que su introducción haya significado un replanteamiento del modelo pedagógico (García-Valcárcel, Basilotta y López, 2014; Medina y Ballano, 2015; Ornellas, Sánchez, Fraga y Domingo, 2015). Y aquí llegamos a la pregunta clave: ¿por qué, a pesar de disponer de herramientas tecnológicas, se mantienen muchas de las prácticas pedagógicas anteriores al siglo XXI?

Aplicamos la fórmula utilizada en las políticas educativas en torno a las TIC: A (equipamiento tecnológico) $+\mathrm{B}$ (formación docente) $=\mathrm{C}$ (cambio educativo). Los estudios nos demuestran que A pero no C. Entonces, siguiendo la lógica deductiva, tampoco B. Diferentes autores e informes (González y De Pablos, 2015; Somekh, 2008) señalan la formación y la falta de confianza del profesorado como un factor explicativo de la dificultad de integrar las TIC en las pedagogías educativas. En este sentido, disponemos de varios estudios recientes en nuestro país (Pérez y Rodríguez, 2016; Fernández-Cruz y Fernández-Díaz, 2016; Roig-Vila, Mengual-Andrés y Quinto-Medrano, 2015) que corroboran la falta de formación instrumental y pedagógica en el uso de las TIC por parte de los maestros. Las creencias y actitudes de los docentes hacia estas herramientas (y la utilidad que tienen en el ámbito educativo) se consideran otro factor esencial (Inan y Lowther, 2010; Tirado-Morueta y Aguaded-Gómez, 2014). De todo lo anterior se desprende que para la integración y uso pedagógico de las TIC deben darse, entre otros, tres condicionantes principales: infraestructura, formación -en aspectos tecnológicos y metodológicos de integración de las TIC - y una actitud favorable hacia los recursos. En otras palabras, han de cubrirse las dimensiones material, cognitiva y afectiva y/o actitudinal. Poder, saber y querer.

Hasta ahora, las políticas se han dirigido a abastecer las aulas de recursos tecnológicos. En adelante, estas deberían centrarse en dar una formación integral a los maestros. Una formación en habilidades y conocimientos, pero también una formación centrada en las actitudes. Porque el simple conocimiento no mueve a la acción. Como sostiene R. Pérez (2006), «es necesario un conocimiento profundo, atravesado de la dimensión afectiva, emocional, capaz de hacer atractivo y deseable su objeto y, por lo tanto, de situarse a las puertas de la acción, del comportamiento» (p. 31).

El Programa Escuela 2.0 no ha generado cambios sustantivos en las metodologías de aula. Pero menos incidencia ha tenido en la comunicación entre maestros y familias (Area y Sanabria, 2014). Antes de la entrada del nuevo siglo, algunos autores (Bauch, 1998; Bernstein, 1998) ya apostaban por el uso de las TIC para mejorar la comunicación y relación entre progenitores y docentes. En los últimos años, los estudios internacionales en este campo se han multiplicado (Bouffard, 2008; Goodall, 2016; Heath, Maghrabi y Carr, 2015; Hohlfeld, Ritzhaupt y Barron, 2010; Lewin y Luckin, 2010; Rogers y Wright, 2008; Thompson, 2008). Y en España, las pocas investigaciones que tenemos, versan tanto sobre el uso de las TIC para la comunicación entre padres y maestros (Ballesta y Cerezo, 2011; Beneyto-Seoane, Collet-Sabe, Cortada-Pujol y Sánchez-Garrote, 2013; Sánchez-Garrote y Cortada-Puyol, 2015) como para mejorar la participación familiar en las instituciones educativas (Vázquez, López y Colmenares, 2014). Se trata, pues, de una línea de estudio emergente, cuya relevancia se apoya en 
las ventajas que ofrecen las TIC para mejorar la relación entre ambos agentes: eficiencia, eficacia, rapidez, efectividad, conveniencia y supresión de barreras temporales (Bernstein, 1998; Olmsted, 2013).

No obstante, los correos electrónicos y las plataformas digitales han tenido poca aceptación en las instituciones educativas (Macià, 2016; Thompson, 2008). La escasez de tiempo del profesorado, los problemas de conectividad en los centros y la poca formación en TIC de los maestros son posibles factores que obstaculizan el uso de estos recursos (Beneyto et al., 2013; Heath et al., 2015; Palomares, 2015). Si nos fijamos en las familias, Lewin y Luckin (2010) y Rogers y Wright (2008) citan las siguientes barreras: una actitud acomodaticia con los actuales canales de comunicación, la falta de acceso y la falta de formación. Se constata así la brecha digital como uno de los posibles obstáculos para el uso de las TIC en la relación familia-escuela, entendiendo por brecha digital la situación causada por desigualdades estructurales -donde por problemas económicos o sociales no se tiene acceso a las tecnologías- o por desigualdades de agencia -cuando se tiene acceso pero no se sabe qué hacer con ellas (Ortoll, Casacuberta y Collado, 2007). Existen investigaciones que prueban la escasa accesibilidad de ciertas familias a los recursos tecnológicos (Bouffard, 2008; Heath et al., 2015; Segura y Martínez, 2011). Otros autores (Sánchez-Garrote y Cortada-Puyol, 2015), en cambio, sostienen y demuestran que la brecha digital emana, sobre todo, de la falta de conocimientos y habilidades por parte de las familias. Es más, en muchas ocasiones, los progenitores son conscientes de sus limitaciones en el manejo y uso social de las TIC y piden formación al respecto. Así lo demuestran en sus estudios Ballesta y Cerezo (2011) y Segura y Martínez (2011).

La realidad descrita nos sitúa ante un reto importante para las políticas educativas: la alfabetización digital de toda la comunidad. Y dentro del concepto de alfabetización digital incluimos la adquisición de habilidades y actitudes relacionadas con la búsqueda, comprensión, creación y comunicación de productos e informaciones utilizando las tecnologías. Es decir, para mejorar la relación familia-escuela mediada por TIC, docentes y familias deberían formarse en los cinco ámbitos competenciales de la alfabetización digital, que siguiendo a Area y Pessoa (2012) son los siguientes: competencias instrumentales (habilidades de manipulación del hardware y el software informático), competencias cognitivas (aprender a usar la información tanto para acceder como para reconstruirla personalmente), competencias socio comunicativas (desarrollo de comportamientos que permitan establecer comunicaciones fluidas y positivas hacia los demás), competencias axiológicas (evitar conductas de comunicación socialmente negativas) y competencias emocionales (de control de las emociones negativas que puedan aparecer con el uso de las TIC).

Partiendo de las investigaciones previas en nuestro campo de estudio, y a la luz de los informes que argumentan la lenta incorporación pedagógica de las TIC, consideramos la falta de acceso y el analfabetismo digital de algunas familias y docentes dos factores que podrían influir negativamente en el uso comunicativo de las TIC. Contemplamos también las actitudes de ambos agentes como un tercer factor a tener en cuenta. La investigación que presentamos tiene como objetivo analizar los discursos de docentes y familias y comprobar si estos tres factores juegan un papel determinante en las nuevas relaciones digitales entre ambos agentes educativos. 


\section{Método}

Este artículo forma parte de una investigación más amplia sobre la participación de las familias en los centros educativos, cuyo objetivo principal es 1) conocer qué y cómo participan las familias en las instituciones escolares y 2) explorar los discursos de los agentes implicados (docentes y progenitores) en torno a tal realidad participativa. Para ello, el método etnográfico se presenta como el más adecuado, ya que se utiliza mayoritariamente en las investigaciones que tienen por objeto de estudio cuestiones descriptivas e interpretativas como son los valores, las ideas y las prácticas de grupos culturales concretos (Poupart, 2010; Robertt y Lisdero, 2016). Así pues, el trabajo de campo, que se ha realizado a lo largo de los cursos escolares 2013-2014 y 2014-2015 (de abril a noviembre de 2014), ha combinado diferentes técnicas de recogida de información: observación participante y no participante, conversaciones informales, entrevistas semiestructuradas y análisis de documentos y páginas web. No obstante, para este artículo se han utilizado solamente las entrevistas a docentes y familiares, ya que es a través de los discursos de los implicados que podemos conocer los condicionantes principales del uso -o no- de las TIC para la comunicación familia-escuela. Concretamente, los objetivos del presente texto son: 1) identificar los factores asociados al uso comunicativo de los recursos digitales y 2) determinar si los tres condicionantes para el uso pedagógico de las TIC -poder, saber y querer- también pueden considerarse los tres elementos a partir de los cuales establecer líneas de actuación que incrementen el uso de estas nuevas herramientas para la relación familia-escuela.

\section{Muestra}

La muestra está formada por 20 centros de educación primaria situados en cuatro comunidades autónomas: Aragón, Cataluña, La Rioja y Las Islas Baleares. La selección de estas comunidades autónomas tiene una doble motivación: de equipo y de voluntad de superar el ámbito territorial autonómico, tratándose además de autonomías con perfil diverso respecto a las políticas educativas, a los programas de implicación familiar, a la presencia de alumnado de origen extranjero en sus aulas (así como políticas diferentes de incorporación de este alumnado y sus familias) o a la existencia de una segunda lengua oficial, entre otras. Respecto al equipo, este está formado por investigadores de las Universidades de Lleida, Girona, Las Islas Baleares, Zaragoza y La Rioja, que han trabajado de forma conjunta durante años y que además son profundamente conocedores de sus respectivas comunidades, hecho que aportaba una situación de partida privilegiada.

Las escuelas de primaria seleccionadas lo fueron por criterios de tamaño (escuelas rurales y urbanas), localización (distribuidas por todo el territorio dentro de cada comunidad autónoma), tipología (escuelas públicas y concertadas) y perfil de alumnado (diferentes porcentajes de inmigración). Además de que todas ellas contaran con indicadores de calidad respecto a la participación de las familias (que fueran comunidades de aprendizaje o contaran con experiencias de éxito reconocidas, premios, etc.). Estos indicadores se detectaron en una fase previa de entrevistas en 
profundidad a 46 personas representantes de las administraciones educativas, de Consejos Escolares Autonómicos, de Federaciones de Asociaciones de Madres y Padres de Alumnado, de representantes sindicales y de movimientos de renovación pedagógica, etc. (para ampliar el perfil de estas entrevistas previas se puede consultar, Garreta Bochaca, 2017). Una vez detectados los centros que respondían al citado perfil, y tomando como punto de partida obtener una muestra en la que hubiera centros rurales y urbanos, públicos y privados, y con diferentes perfiles de alumnado, en cada comunidad se decidió estudiar: una escuela rural, un centro concertado, uno público, una comunidad de aprendizaje y un centro con un elevado porcentaje de inmigración. La decisión de contar con las tipologías de escuela ha estado motivada por la voluntad del equipo investigador de conocer qué discursos y prácticas participativas son comunes a la mayoría de centros de educación primaria y cuáles son características de un determinado modelo de escuela, con el consiguiente hándicap de representatividad que este tipo de muestras implican.

\section{Instrumentos}

Como se ha apuntado en el apartado introductorio de la metodología, para este artículo se han utilizado las entrevistas a docentes y familiares dado que esta técnica permite comprender los discursos y puntos de vista subjetivos de los entrevistados (Flick, 2014) y por lo tanto, se presenta como el instrumento idóneo para alcanzar los objetivos del estudio: analizar los discursos de familias y maestros para detectar posibles barreras o estímulos al uso de las TIC en la comunicación familia-escuela.

El guion de la entrevista empleada para la recogida de información, de carácter semi-estructurado, fue elaborado por los investigadores del proyecto a partir del marco teórico de partida y de las citadas entrevistas previas, validándose posteriormente su idoneidad con estos mismos entrevistados (considerados expertos en la temática, quienes enriquecieron los puntos a tratar y los enfoques). Finalmente, el guion consistió en 6 grandes bloques temáticos, siguiendo las modalidades de participación familiar que plantea Epstein (1992): crianza, comunicación, voluntariado, aprendizaje en casa, toma de decisiones y colaboración con la comunidad. En relación al ámbito de la comunicación familia-escuela, se establecieron tres sub-temáticas a tratar en las entrevistas: 1) Los canales de comunicación tradicionales: uso y valoración personal; 2) Los canales de comunicación tecnológicos unidireccionales: uso y valoración; y 3) Los canales de comunicación tecnológicos bidireccionales: uso y valoración. Es importante destacar también que en la investigación no se preguntó directamente por los tópicos que aquí analizamos (acceso, formación y actitudes a favor o en contra), sino que estos aparecieron en los discursos de los informantes al tratar la cuestión de la comunicación en las escuelas. Este hecho aporta un valor añadido al artículo que presentamos, ya que el estudio muestra las inquietudes reales de familiares y docentes hacia los canales de comunicación tecnológicos (al no tratarse de cuestiones directamente demandadas por los entrevistadores en las sub-temáticas 2 y 3 antes mencionadas).

En total, se han entrevistado un mínimo de diez informantes en cada uno de los centros, siguiendo también criterios específicos en la selección de los mismos. Concretamente, se ha garantizado una equidad numérica entre familias y personal 
docente, de forma que se recogiera la opinión de docentes (un maestro de cada ciclo, el director y otra persona con responsabilidad en el centro, es decir, un mínimo de cinco) y el mismo número de familiares con diferentes perfiles: miembros activos/ no activos de la junta de las AMPA, representantes/no representantes del Consejo Escolar, y padres y madres cuyos hijos cursaran diferentes niveles educativos.

Las entrevistas, de duración entre 60 y 90 minutos, se han realizado dentro de las escuelas estudiadas, en las universidades participantes o en espacios privados que permitieran su desarrollo sin interrupciones. Al inicio de las entrevistas, los investigadores recordaron a los informantes el objetivo de la entrevista y los puntos más importantes que se iban a tratar, así como el compromiso ético de todos los investigadores de garantizar el total anonimato de las personas entrevistadas, así como de los sujetos sobre los que pudiera versar la conversación. Todas las entrevistas fueron grabadas y se transcribieron también en su globalidad.

\section{Procedimiento}

En todas las entrevistas (realizadas a 200 informantes) se han seleccionado los fragmentos de las mismas que tratan la cuestión del uso y valoración de las TIC en la comunicación unidireccional y bidireccional entre familia y escuela (sub-temáticas 2 y 3 del ámbito de la comunicación familia-escuela). A continuación se ha llevado a cabo un análisis de contenido, que se ha focalizado en los discursos de docentes y familiares en torno al acceso, la formación y las actitudes que tienen respecto a las TIC. Para ello, se ha realizado un análisis exhaustivo de cada una de las entrevistas y se han extraído los fragmentos relacionados con los tópicos expuestos, llevando a cabo un análisis etic de la información, es decir, la codificación de las entrevistas a partir de categorías resultantes de la literatura previa (Maxwell, 2005). Una vez finalizada esta primera criba de información, se han codificado 30 entrevistas para establecer las primeras sub-categorías. Se ha utilizado el mismo esquema de codificación para analizar el resto de entrevistas con el fin de asegurar una consistencia analítica. Posteriormente, se han agrupado todas las sub-categorías en significados similares. El resultado de este proceso ha sido la emergencia de cuatro categorías generales a partir de las cuales se ha organizado toda la información: hábito de uso, acceso, formación y preferencia por los canales tradicionales.

\section{Resultados}

El primer dato reseñable es que los discursos en torno al acceso, la formación y las actitudes hacia los canales de comunicación tecnológicos (conceptualizados en las categorías de «hábito de uso», «acceso», «formación» y «preferencia por los canales tradicionales») aparecen reiteradamente en las entrevistas (y en todos los perfiles de centro analizados). Y, sobre todo, los encontramos en los relatos sobre correos electrónicos y plataformas digitales (en las declaraciones sobre páginas web y blogs hay pocas referencias a las ideas que analizamos). Ahora bien, estos resultados, lejos de poder considerarse azarosos, nos sitúan ante el segundo dato a destacar: los discursos de accesibilidad, formación, hábito y preferencia por los medios tradicionales se relacionan principalmente con las herramientas digitales cuya implementación está poco 
generalizada en las instituciones educativas. Todos los centros analizados, excepto uno, están presentes en la red a través de páginas web o blogs. Pero solamente tres escuelas utilizan las plataformas para informar y comunicarse con las familias, el mismo número que se sirve del correo electrónico para idéntico fin (Macià, 2016). Coincide, pues, la aparición o no de los relatos que estudiamos con el grado de utilización de los canales digitales para la relación familia-escuela (a menor frecuencia de uso del medio, mayor número de discursos que aluden a los tópicos que abordamos). Por consiguiente, hemos centrado el análisis en los correos electrónicos y las plataformas digitales (bloque temático 3 de la entrevista). De las 200 entrevistas, solamente 65 (30 familias y 35 docentes de 11 escuelas diferentes), abordaban la cuestión comunicativa de las TIC en profundidad, declarando el resto de los entrevistados que ellos no utilizaban las herramientas tecnológicas para comunicar y que por lo tanto, no podían valorarlas ni opinar al respecto. Así pues, situados en las 65 entrevistas que profundizan en los discursos de la comunicación bidireccional utilizando las TIC, encontramos 48 entrevistas que contienen referencias a alguna de las cuatro categorías establecidas, lo que representa el 73\% del total de entrevistas tomadas en consideración para el análisis.

En el citado contexto, todos los informantes consideran a las familias como las más expuestas a sufrir desigualdades de acceso, de formación y, consecuentemente, de hábito. Los entrevistados nombran en 43 ocasiones estos aspectos cuando hablan de las familias, mientras que la frecuencia de aparición de estos tópicos en alusión a los docentes es mucho menor: 9. Si analizamos por separado cada una de estas categorías, la idea que señalan con más frecuencia padres y maestros es la relativa a las desigualdades de acceso en los hogares. El 36\% de los entrevistados destacan la falta de equipamiento y/o conexión que sufren algunas familias en la mayoría de los centros. En menor medida aparece el discurso referente al hábito, nombrado por el 18\% de los informantes. Y, en tercer lugar, se sitúa la necesidad de formación de los progenitores -idea expuesta solamente por el 10\% de la población estudiada. En el terreno de las actitudes, ambos colectivos muestran por igual una preferencia por los canales tradicionales. Y, además, lo hacen con frecuencias elevadas. Esta categoría aparece en 25 de las 67 entrevistas, concretamente en 12 docentes y 13 familiares. En la tabla 1 se recogen las frecuencias obtenidas para cada una de las categorías según colectivo.

Tabla 1

Frecuencia de aparición de las categorías en las entrevistas

\begin{tabular}{lccc}
\hline Categorías & \multicolumn{3}{c}{ Frecuencias } \\
\hline & En los docentes & En las familias & Total \\
\hline Poco hábito en el uso de las TIC & 3 & 12 & 15 \\
Desigualdades de acceso & 4 & 24 & 28 \\
Falta de formación & 2 & 7 & 9 \\
Preferencia por canales tradicionales & 12 & 13 & 25 \\
\hline Total & 21 & 56 & 77 \\
\hline
\end{tabular}




\section{Poco hábito en el uso de las TIC}

Algunas familias consideran que los recursos digitales no forman parte de la cotidianidad profesional de ciertos docentes, lo que supone una barrera importante en la introducción de las TIC para la comunicación y relación con ellos. No obstante, la idea que prevalece entre padres y maestros es la falta de hábito por parte de las familias. Este discurso emerge en nueve entrevistas y otros tres informantes, aun siendo conscientes de que hay padres y madres que no utilizan con asiduidad los recursos digitales, creen que cada vez hay muchos más hogares en los que las TIC ocupan un lugar importante en la vida diaria de sus miembros. Destacamos algunas referencias sobre esta cuestión:

«Hay maestros que son más activos y otros que no tanto pero, bueno, en general, contestan.» (Representante AMPA, centro concertado, Cataluña). «La gente, hablamos de los inmigrantes, pero nosotros tampoco miramos los e-mails. La gente no los mira. A mí me sorprende, porque yo miro el correo cada día. Siempre. Y me estoy dando cuenta de que no todo el mundo mira el correo cada día, de que no es tan habitual entre las familias.» (Familiar, centro concertado, Cataluña).

\section{Desigualdades de acceso}

Las escuelas no manifiestan problemas de acceso a la red. Solamente en tres centros de Aragón los docentes consideran los problemas de conectividad como una barrera en la comunicación con las familias. Ambos agentes, en cambio, están preocupados por las desigualdades estructurales que existen en los hogares. Uno de los centros con mucha inmigración no utiliza ninguna herramienta TIC para relacionarse con los progenitores debido al elevado número de familias sin posibilidad de acceder a estos recursos. Y otras cuatro escuelas, a pesar de disponer de página web y/o blog de centro para difundir información, no se sirven de los canales tecnológicos para la comunicación bidireccional con las familias. Según los maestros de estas cinco instituciones, un cambio de rumbo en los medios utilizados podría perjudicar el fin al que sirven: facilitar el flujo de mensajes entre emisor-receptor y viceversa. La brecha digital entre las familias se concibe, pues, como el principal motivo por el que se siguen utilizando solamente los canales tradicionales.

Asimismo, los docentes de los centros que sí se comunican con los padres a través de correos electrónicos o plataformas, también conciben las desigualdades de acceso entre las familias como un problema que aún persiste en la mayoría de comunidades educativas. En estas escuelas, los profesionales son conscientes de la existencia de múltiples y variadas situaciones sociales y económicas, y de vivir en una realidad compleja ante la cual adoptan una solución: combinar los canales tecnológicos con los medios de comunicación más tradicionales (tutorías, reuniones grupales, encuentros cara a cara, circulares y agenda). Las familias, aunque en menor medida que los maestros, también aluden a las desigualdades de acceso en sus discursos. Mostramos algunas declaraciones que evidencian estas concepciones y actitudes: 
«Nosotros tenemos un periódico del colegio, un blog. Pero, desde luego, las cuestiones de comunicaciones, de avisos y tal... no. Nosotros utilizamos, para casi todo, el boca a boca. Para empezar, muchas familias de aquí tenemos un alumnado socialmente desfavorecido, no tienen ordenador.» (Director, centro alta inmigración, Aragón).

«Internet no tiene todo el mundo. En nuestro colegio, la realidad que hay es que no llega a todos. Entonces, te tienes que asegurar de que llegue por agenda por lo menos. Aunque pongas en la plataforma una información, por ejemplo, los deberes, nunca irá solo por la red, va por otras vías.» (Docente, centro concertado, Aragón).

\section{Falta de formación}

Los discursos relativos al segundo nivel de exclusión digital son menos importantes. Solamente dos maestros aseguran no disponer de la formación adecuada para utilizar los canales digitales en su quehacer laboral diario - una falta de formación que relacionan con la edad. Esta carencia competencial personal también la manifiestan tres familiares. Asimismo, hay docentes que perciben la falta de habilidades y conocimientos TIC por parte de los progenitores como un factor que obstaculiza la implementación y uso de los e-mails y las plataformas para mejorar la relación familia-escuela. Algunos ejemplos que exponen la falta de formación en ambos colectivos:

«Yo soy una persona que, por edad y por formación, soy un advenedizo en esto.» (Director, centro concertado, La Rioja).

«No me parece mal que se vayan introduciendo, pero me parece que tenían que haberse dado unos cursos. Se explicó, pero no pienso que todos los padres estén preparados para ese funcionamiento digital.» (Docente, escuela rural, La Rioja).

En este punto debemos exponer una limitación del estudio. Las desigualdades de «agencia» ante los medios digitales pueden producirse por falta de conocimiento técnico, pero también por falta de destrezas centradas en el uso social de la información y la comunicación. El análisis de contenido desvela que algunos informantes se refieren claramente a las habilidades puramente instrumentales. No obstante, hay entrevistados que no especifican a qué tipo de formación se refieren cuando aluden a la misma, lo que nos obliga a hablar simplemente de formación -sin desgranar si se trata de formación puramente instrumental o de competencias en el uso social y comunicacional de las TIC.

\section{Preferencia por canales tradicionales}

La preferencia por los canales tradicionales aparece como el cuarto discurso a valorar en el establecimiento de líneas de actuación para favorecer el uso comunicativo de las TIC. Maestros y familias impregnan sus declaraciones de argumentos distintos, pero todos ellos formulados desde la desconfianza o inseguridad hacia la capacidad comu- 
nicativa de los nuevos recursos. Algunos docentes, todos ellos ubicados en centros que usan los e-mails o las plataformas, muestran la voluntad de utilizar estas herramientas para ciertas informaciones (académicas y/o puntuales) y dejar el contacto directo para cuestiones complejas y delicadas que requieran de más tiempo y de una relación más personal. Los maestros también nombran la agenda y las circulares en sus declaraciones de preferencias, así como los carteles y la comunicación a través de los niños. En estos casos se trata mayoritariamente de maestros que ejercen su profesión en escuelas que no utilizan cotidianamente los canales digitales.

Si nos centramos en las familias, estas también optan, en gran medida, por los medios tradicionales. Y en este caso, el discurso que más se repite es el que alaba la comunicación directa y el contacto cara a cara. Otros progenitores argumentan su preferencia por los canales tradicionales a partir de alusiones positivas a las circulares y a la agenda. Ahora bien, ¿qué tipo de familias expone este discurso de preferencia hacia los medios tradicionales? El primer dato interesante es que no se trata de un perfil homogéneo, sino que se da en progenitores cuyos hijos cursan distintas etapas educativas y con diferentes niveles de implicación en las AMPA. Ahora bien, como sucede con el colectivo docente, la diferencia estriba en el enfoque que los entrevistados dan a sus palabras cuando exponen tal cuestión. En las escuelas que utilizan los e-mails o las plataformas, los discursos de las familias se construyen en torno a dos ideas principales: la limitación del uso de las TIC a ciertas informaciones rápidas y puntuales y la exposición de algún atributo negativo de la herramienta a partir de la propia experiencia. En los centros que optan por los canales tradicionales, los progenitores tienen una actitud más taxativa hacia el uso los medios digitales alegando que son innecesarios o inadecuados para el centro de sus hijos -por ser pequeño o por tratarse de la educación primaria. En relación con esta cuestión, familias y docentes lo expresan así:

«Por una parte viene bien, pero, personalmente, me gusta el trato más cercano, en este sentido. No es lo mismo hablar a una máquina... es un poco más frío.» (Docente, centro concertado, La Rioja).

«La agenda es mucho más inmediata. Si me la firman, al día siguiente la tengo.» (Docente, centro concertado, Aragón).

\section{Discusión y conclusiones}

Numerosos estudios (Area y Sanabria, 2014; García-Valcárcel et al., 2014; Medina y Ballano, 2015) demuestran que el proyecto 2.0 y otros programas similares han servido para mejorar la dotación de ordenadores y la conexión en los centros, una realidad que se evidencia también en el contexto que estudiamos. Solamente en tres centros persiste una preocupación docente por el mal funcionamiento de la red en determinadas escuelas (se trata, sobre todo, de edificios con mucha antigüedad). En cuanto a las familias, el estudio también muestra los resultados obtenidos en otras investigaciones (Heath et al., 2015; Segura y Martínez, 2011): la falta de acceso de este colectivo es un problema social que persiste en el siglo XXI. Progenitores y maestros son conscientes de la importancia que tiene la dimensión estructural en las situaciones de exclusión 
digital, como se pone de manifiesto en las múltiples referencias a ello -en total, un tercio de los entrevistados. Por lo tanto, el acceso general de las familias a las TIC es una cuestión pendiente y a la cual debemos prestar atención cuando se implementen los recursos tecnológicos para la relación con las familias.

A diferencia de los resultados obtenidos en los centros analizados, Rogers y Wright (2008) no evidenciaron importantes diferencias de acceso a las TIC entre familias de distintos niveles socioculturales. Las desigualdades, según ellos, estriban en el uso que se hace de los nuevos recursos para la información y comunicación con las escuelas. En España también encontramos autores que respaldan esta idea (Ballesta y Cerezo, 2011; Segura y Martínez, 2011). Y, en nuestro caso, el discurso de la falta de hábito de algunas familias, aparecido en doce ocasiones, también apoya la existencia de desigualdades en el uso de las TIC expuesta por Rogers y Wright (2008). No obstante, la frecuencia de este relato en la presente investigación ha sido menor que el referente al acceso (lo que nos lleva a concluir que la accesibilidad es un factor más relevante para los agentes educativos implicados en el estudio). Asimismo, los datos recogidos no nos permiten determinar las causas de la diferenciación de uso según familias, pero sí que podemos inferir que la formación y las actitudes son dos de las variables explicativas del hecho que analizamos.

Por lo que se refiere a la falta de habilidades y competencias TIC de las familias, se trata del discurso menos nombrado por los informantes. Además, solamente tres padres reconocen no utilizar las tecnologías por falta de conocimientos. La formación de los maestros tampoco es una cuestión que preocupe demasiado a ambos agentes educativos. Beneyto et al., (2013), de forma similar, exponen que la falta de formación docente es una limitación en el uso de las TIC, pero sin determinar la fuerza de influencia que este factor ejerce en las relaciones entre padres y maestros. En cuanto a las actitudes, como ya demuestran algunos estudios (Rogers y Wright, 2008; Thompson, 2008), muchos maestros y progenitores entrevistados siguen mostrando una preferencia por los canales tradicionales, lo que convierte este discurso en un factor potencial en el estudio de los recursos digitales para la comunicación familia-escuela. Y, en este caso, ya no se trata de una cuestión que afecte sobre todo a las familias -como sucede con los tres discursos anteriores-, sino que ambos agentes, en un elevado porcentaje, son partidarios de mantener relaciones con el «otro» a través de los medios ya existentes. Ahora bien, ¿por qué se siguen prefiriendo los medios tradicionales? ¿Qué inconvenientes perciben en las herramientas TIC? ¿Qué ventajas les ofrecen los canales tradicionales versus los digitales? Futuras investigaciones deberían analizar y comprender los factores subjetivos y actitudinales que inhiben, aún hoy en día, el uso de la tecnología para la comunicación familia-escuela. Asimismo, atendiendo a las limitaciones de representatividad del estudio que presentamos, deberían llevarse a cabo nuevas investigaciones con amplias muestras de población que permitieran determinar si los factores que aquí se plantean (acceso, formación y actitudes) son realmente determinantes en el uso de las TIC en la relación familia-escuela, y qué peso ejerce cada una de estas variables en los comportamientos comunicativos de ambos agentes.

No obstante, los resultados del presente estudio nos llevan a una primera conclusión: el acceso de las familias, la formación de ambos agentes y, sobre todo, el cambio 
de actitudes por parte de los dos colectivos son tres posibles condicionantes para que las relaciones entre familias y docentes mediadas por TIC puedan implementarse de forma exitosa en los centros. Por lo tanto, y cumpliendo el segundo objetivo que nos planteamos, se constata que los estudios anteriores sobre el uso de las TIC en el aula pueden servirnos de inspiración y de conocimiento científico a partir del cual empezar a dibujar las hipótesis y preguntas que guíen la investigación en nuestro campo.

Acceso y alfabetización se constituyen, pues, como las dos líneas de actuación que deberían seguir las políticas y las comunidades educativas si se quiere aprovechar el potencial de las TIC para mejorar la relación entre ambos agentes, como apuntan también otros estudios a nivel internacional (Bouffard, 2008; Rogers y Wright, 2008; Thompson, 2008). Ahora bien, la presente investigación aporta una nueva luz a los informes anteriores y amplía la necesidad de alfabetización digital también a los docentes -centrada, principalmente, en el cambio de actitudes hacia los nuevos canales de comunicación. Por lo tanto, cuando hablamos de alfabetización digital para mejorar la relación familia-escuela hacemos referencia al resultado de una educación integral. De una educación que se sitúe más allá de la formación en los cinco ámbitos competenciales de la alfabetización digital (Area y Pessoa, 2012) y ayude a crear actitudes positivas y abiertas hacia los recursos. De una educación cuyo objetivo sea construir individuos conscientes de los beneficios que aportan los nuevos recursos; individuos capaces de escoger el mejor medio para cada momento y cada necesidad; individuos, simplemente, alfabetizados de manera íntegra en la nueva cultura digital. Para lograrlo, la formación permanente del profesorado debería mirar también hacia la relación familia-escuela y, dentro de esta, hacia las nuevas posibilidades que ofrecen las TIC para mejorar la comunicación entre ambos. Además, las escuelas deberían ser las encargadas de abrir sus puertas y adquirir un papel fundamental como agentes de alfabetización del resto de la comunidad educativa (Ballesta y Cerezo, 2011), contribuyendo así a mejorar la relación familia-escuela y al mismo tiempo, a formar ciudadanos cultos y capaces de desarrollarse plenamente en la nueva sociedad digital.

\section{Referencias}

Area, M., Alonso, C., Correa, J.M., Del Moral, M.E., De Pablos, J., Paredes, J., \& Valverde, J. (2014). Las políticas educativas TIC en España después del Programa Escuela 2.0: las tendencias que emergen. RELATEC. Revista Latinoamericana de Tecnología Educativa, 13(2), 11-33. doi: http://dx.doi.org/10.17398/1695-288X.13.2.11

Area, M., \& Pessoa, T. (2012). De lo sólido a lo líquido: las nuevas alfabetizaciones ante los cambios culturales de la Web 2.0. Comunicar, 38(XIX), 13-20. doi: https:// doi.org/10.3916/C38-2012-02-01

Area, M., \& Sanabria, A. (2014). Opiniones, expectativas y valoraciones del profesorado participante en el Programa Escuela 2.0 en España. Educar, 50(1), 15-39. doi: http:// dx.doi.org/10.5565/rev/educar.64

Ballesta, J., \& Cerezo, M.C. (2011). Familia y escuela ante la incorporación de las tecnologías de la Información y la Comunicación. Educación XX1, 14(2), 133-156. doi: http://dx.doi.org/10.5944/educxx1.14.2.248 
Bauch, J.P. (1998). Applications of technology to linking schools, families, and students. Proceeding of the Families, Technology, and Education Conference. Recuperado de http:// goo.gl/sk9ntp.

Bauman, Z. (2003). Modernidad Líquida. México: Fondo de Cultura Económica.

Beneyto-Seoane, M., Collet-Sabe, J., Cortada-Pujol, M., \& Sánchez-Garrote, I. (2013). ¿Mucho ruido y pocas nueces? Escuelas, familias y TIC: discursos, realidades, logros y retos. In D. Aranda, A. Creus, \& J. Sanchez-Navarro (Eds.), Educación, medios digitales y cultura de la participación (pp.241-262). Barcelona: Editorial UOC.

Bernstein, A. (1998). Using electronic mail to improve school-based communications. THE Journal. Recuperado de https://goo.gl/bBI5m2.

Bouffard, S.M. (2008). Tapping into technology: The role of the Internet in family-school communication. Cambridge, MA: Hardvard Family Research Project. Recuperado de http://goo.gl/bGi1ke.

Brey, A., Innerarity, D., \& Mayos, G. (2009). La Sociedad de la Ignorancia y otros ensayos. Barcelona: Zero Factory, S.L. Recuperado de http://goo.gl/61YAlV.

Burch, S. (2005). Société de l'information et Société de la connaissance. In V. Peugeot \& A. Ambrosi (Eds.), Enjeux de mots. Regards multiculturels sur les sociétés de l'information (pp.49-71). France: C\&F éditions.

Castells, M. (2006). La Sociedad red: una visión global. Madrid: Alianza Editorial.

Epstein, J. (1992). School and family partnerships. Report nº 3. Center on Families Communities, Schools and Children's Learning. Baltimore, Md.: johns hopkins Univ.

Fernández-Cruz, F.J., \& Fernández-Díaz, M.J. (2016). Los docentes de la Generación $\mathrm{Z}$ y sus competencias digitales. Comunicar, 46(XXIV), 97-105. doi: http://dx.doi. org/10.3916/C46-2016-10

García-Valcarcel, A., Basilotta, V., \& López, C. (2014). Las TIC en el aprendizaje colaborativo en el aula de Primaria y Secundaria. Comunicar, 42(XXI), 65-74. doi: http://dx.doi.org/10.3916/C42-2014-06

Garreta Bochaca, J. (coord.) (2017). Familias y escuelas. Discursos y prácticas sobre la participación en la escuela. Madrid: Pirámide.

González, N., Gozálvez, V., \& Ramírez, A. (2014). La competencia mediática en el profesorado no universitario. Diagnóstico y propuestas formativas. Revista de Educación, 367, 117-146. doi: http://dx.doi.org/10.4438/1988-592X-RE-2015-367-285

González, A., \& De Pablos, J. (2015). Factores que dificultan la integración de las TIC en las aulas. Revista de Investigación Educativa, 33(2), 401-417. doi: http://dx.doi. org/10.6018/rie.33.2.198161

Goodall, J.S. (2016). Technology and school-home communication. International Journal of Pedagogies and Learning, 11(2), 118-131. doi: http://dx.doi.org/10.1080/22040552.2 016.1227252

Heath, D., Maghrabi, R., \& Carr, N. (2015). Implications of Information and Communication Technologies (ICT) for school-home communication. Journal of Information Technology Education: Research, 14, 363-396. Recuperado de http://goo.gl/ngilqs.

Hohlfeld, T., Ritzhaupt, A., \& Barron, A. (2010). Connecting schools, community and family with ICT: Four-year trends related to school level and SES of public schools in Florida. Computers $\mathcal{E}$ Education, 55, 391-405. doi: http://dx.doi.org/10.1016/j. compedu.2010.02.004 
Inan, F., \& Lowther, D. (2010). Laptops in the K-12 Classrooms: Exploring Factors Impacting Instructional Use. Computers $\mathcal{E}$ Education, 55(3), 937-944. doi: http://dx.doi. org/10.1016/j.compedu.2010.04.004

Lee, A., \& So, C. (2014). Alfabetización mediática y alfabetización informacional: similitudes y diferencias. Comunicar, 42(XXI), 137-146. doi: http://dx.doi.org/10.3916/ C42-2014-13

Lewin, C., \& Luckin, R. (2010). Technology to support parental engagement in elementary education: lessons learned from the UK. Computers E Education, 54, 749-758. doi: http://dx.doi.org/10.1016/j.compedu.2009.08.010

Macià, M. (2016). La comunicación familia-escuela: el uso de las TIC en los centros de primaria. Revista Electrónica Interuniversitaria de Formación del Profesorado, 19(1), 73-83. doi: http://dx.doi.org/10.6018/reifop.19.1.245841

Maxwell, J.A. (2005). Qualitative research design: An interactive approach (2n Ed.). Thousand Oaks, CA: Sage.

Medina, A., \& Ballano, S. (2015). Retos y problemáticas de la introducción de la educación mediática en los centros de secundaria. Revista de Educación, 369, 135-158. doi: http://dx.doi.org/10.4438/1988-592X-RE-2015-369-293

Olmsted, C. (2013). Using technology to increase parent involvement in schools. TechTrends, 57(6), 28-37. doi: http://dx.doi.org/10.1007/s11528-013-0699-0

Ornellas, A., Sánchez, J.A., Fraga, L., \& Domingo, L. (2015). Políticas y prácticas en la formación permanente del profesorado en TIC en Cataluña. Revista Electrónica Interuniversitaria de Formación del Profesorado, 18(3), 83-96. doi: http://dx.doi. org/10.6018/reifop.18.3.190271

Ortoll, E., Casacuberta, D., \& Collado, A. (2007). La alfabetización digital en los procesos de inclusión social. Barcelona: Editorial UOC.

Palomares, A. (2015). Análisis de modelos de comunicación, profesorado-familia, para gestionar conflictos: estudio de la comunidad educativa de Albacete. Pedagogía Social. Revista Interuniversitaria, 25, 277-298. doi: http://dx.doi.org/10.7179/PSRI_2015.25.12

Pérez, R. (2006). La educación de calidad: una responsabilidad compartida. Participación educativa, 1, 27-34. Recuperado de http://goo.gl/x0A5ze.

Pérez, A., \& Rodríguez, M.J. (2016). Evaluación de las competencias digitales autopercibidas del profesorado de educación primaria en Castilla y León. Revista de Investigación Educativa, 34(2), 399-415. doi: http://dx.doi.org/10.6018/rie.34.2.215121

Poupart, J.A (2010). Entrevista de tipo qualitativo: considerações epistemológicas, teóricas e metodológicas. In J. Poupart et al. (Orgs.), A pesquisa qualitativa: enfoques epistemológicos e metodológicos (pp.215-253). Petrópolis, RJ: Vozes.

Robertt, P., \& Lisdero, P. (2016). Epistemología y metodología de la investigación sociológica: reflexiones críticas de nuestras prácticas de investigación. Sociologias, 18(41), 54-83. doi: http://dx.doi.org/10.1590/15174522-018004103

Rogers, R. H., \& Wright, V. H. (2008). Assessing technology's role in communication between parents and middle schools. Electronic Journal for the Integration of Technology in Education, 7, 36-58. Recuperado de http://goo.gl/5v7KhP.

Roig-Vila., R., Mengual-Andrés, S., \& Quinto-Medrano, P. (2015). Conocimientos tecnológicos, pedagógicos y disciplinares del profesorado de Primaria. Comunicar, 45(XXIII), 151-159. doi: http://dx.doi.org/10.3916/C45-2015-16 
Sánchez-Garrote, I., \& Cortada-Pujol, M. (2015). Recursos digitales en la relación familia y escuela en la etapa 0-3. Cultura y Educación, 27(1), 221-233. doi: http://dx.doi.org /10.1080/11356405.2015.1006851

Segura, M.L., \& Martínez, M.C. (2011). Análisis del perfil formativo en TIC de padres y madres en relación con su participación en la acción tutorial con la EEPP Sagrada Familia de Linares. Revista Electrónica de Investigación y Docencia, 6, 77-97. Recuperado de http://goo.gl/JdpGkm.

Somekh, B. (2008). Factors affecting teachers' pedagogical adoption of ICT. In J. Voogt \& G. Knezek (Eds.), International Handbook of Information Technology in Primary and Secondary Education (pp.449-460). New York: Springer. Recuperado de http://goo.gl/ujL4hg.

Thompson, B.C. (2008). Characteristics of parent-teacher email communication. Communication Education, 57(2), 201-223. doi: http://dx.doi.org/10.1080/03634520701852050

Tirado-Morueta, R., \& Aguaded-Gómez, J.I. (2014). Influencia de las creencias del profesorado sobre el uso de la tecnología en el aula. Revista de Educación, 363, 230 255. doi: http://dx.doi.org/10.4438/1988-592X-RE-2012-363-179.

Vázquez, E., López, E., \& Colmenares, L. (2014). La dimensión socializadora de la tecnología para una comunidad educativa más abierta y colaborativa. Revista de Tecnología de Información y Comunicación en Educación, 8(1), 145-157. Recuperado de http://goo.gl/ZQazef.

\section{Apéndice. Entrevista a las familias y a los docentes (adaptar las preguntas según el perfil de entrevistado)}

\section{Preguntas generales e introductorias.}

- Descripción del entorno. Características sociodemográficas del barrio/pueblo. ¿Cómo describiría el contexto sociodemográfico en que se ubica el centro?

- Perfil de las familias que acoge el centro. ¿Cómo describiría las familias que acoge el centro?

- Valoración general de la relación entre familias y docentes ¿Cómo se relacionan las familias con el centro y el centro con las familias? ¿Cómo valora esta relación?

- Concepto de participación (en general). ¿Qué entiende usted por participación de las familias en la educación de los hijos?

- ¿Es importante la implicación parental? ¿Favorece el éxito del alumnado? ¿En qué sentido? ¿Qué otros beneficios aporta?

- Introducción a la participación real. ¿En qué participan las familias en este centro?

\section{Crianza.}

A) Acciones de las familias.

- ¿Las familias muestran interés por la educación de sus hijos? 
- ¿Cómo valoraría las normas familiares o hábitos dentro de los hogares? ¿Suficiente, mejorable...?

- ¿Existe algun grupo de familias que necesite más apoyo institucional en este sentido?

B) Acciones del centro.

- ¿Qué se hace desde el centro para ayudar a las familias a crear este ambiente favorecedor?

- ¿Cuál es el papel de los profesionales externos?

- ¿Se llevan a cabo charlas y sesiones informativas para las familias?

\section{Aprendizaje en casa.}

A) Acciones de las familias.

- ¿Las familias se implican en la educación de sus hijos desde casa? ¿Cómo? ¿En qué actividades?

- Guiar al entrevistado en las siguientes actividades: control y ayuda en los deberes, fomento de un ambiente de trabajo en casa, realización de actividades conjuntas entre padres/tutores e hijos...

- ¿Cómo valoraría la implicación de las familias dentro de los hogares? ¿Suficiente, mejorable...?

- ¿Existe algun grupo de familias que necesite más apoyo institucional en este sentido?

B) Acciones del centro.

- ¿Qué se hace desde el centro para ayudar a las familias a mejorar su implicación parental desde los hogares?

- ¿Cuál es el papel de los profesionales externos y de los maestros?

- ¿Se llevan a cabo sesiones informativas para las familias?

\section{Comunicación.}

A) Canales de comunicación tradicionales: uso y valoración.

- ¿Qué canales de comunicación tradicionales utiliza el profesorado para relacionarse con las familias y las familias con el profesorado?

- ¿En qué ocasiones?

- ¿Cómo los valora?

- Si no aparecen, guiar al entrevistado con los siguientes canales: circulares, comunicación informal, agenda, reuniones grupales y tutorías.

B) Canales de comunicación tecnológicos unidireccionales: uso y valoración.

- ¿Cuando el centro quiere comunicar alguna información a todo el colectivo de familias, utiliza algun medio tecnológico?

- ¿En qué ocasiones?

- ¿Cómo lo valora? 
- Si no aparecen, guiar al entrevistado con los siguientes canales: páginas web, blogs, correos electrónicos y plataformas.

C) Canales de comunicación tecnológicos bidireccionales: uso y valoración.

- ¿Se utilizan los emails o las plataformas digitales también para mantener conversaciones con las familias/docentes?

- ¿En qué ocasiones?

- ¿Cómo lo valora?

5. Voluntariado.

A) Actividades festivas y eventos.

- ¿En qué actividades participan las familias?

- ¿Cómo participan?

- ¿Niveles de participación? ¿Quiénes participan?

- ¿Cómo valora esta participación?

- ¿Es importante que las familias participen en este tipo de actividades? ¿Por qué?

- ¿Qué actuaciones lleva a cabo el centro para potenciar esta implicación parental?

B) En el aula.

- ¿En qué actividades participan las familias?

- ¿Cómo participan?

- ¿Niveles de participación? ¿Quiénes participan?

- ¿Cómo valora esta participación?

- ¿Es importante que las familias participen en este tipo de actividades? ¿Por qué?

- ¿Qué actuaciones lleva a cabo el centro para potenciar esta implicación parental?

C) Otras formas de participación en el centro.

- ¿Existen otras formas de participación de las familias en el centro que no hayamos comentado?

- ¿Cómo participan?

- ¿Niveles de participación? ¿Quiénes participan?

- ¿Cómo valora esta participación?

- ¿Es importante que las familias participen en este tipo de actividades? ¿Por qué?

6. Toma de decisiones.

A) El Consejo Escolar .

- ¿Qué decisiones se toman?

- ¿Cuál es el rol de los diferentes asistentes?

- ¿Cómo valoraría el Consejo Escolar? ¿Es útil? 
- ¿Cuál es la implicación de las familias en el mismo?

B) AMPA.

- ¿Qué tipo de familias participan?

- ¿Cuál es la trayectoria del AMPA?

- ¿Cómo ha evolucionado la participación de la misma?

- ¿Cómo se renueva el AMPA y cómo se transmite la información a las familias en estos momentos de renovación?

- ¿Qué actividades organiza el AMPA?

- ¿Cómo son las relaciones con el Equipo Directivo y los profesionales?

- ¿Cuáles diría que son los puntos fuertes y débiles del AMPA y de las actuaciones que realiza?

- ¿Cómo valoraría la participación de las familias en el AMPA?

\section{Colaboración con la comunidad.}

\section{A) Con el entorno.}

- ¿Qué relación se establece con el entorno?

- ¿Se organizan activides con el pueblo/barrio?

- ¿Se colabora con instituciones locales? ¿Cómo?

- ¿Qué beneficios aporta?

B) Con otros profesionales del centro.

- ¿Qué papel tienen los profesionales externos (EAP, trabajadores sociales...) en la relación familia-escuela?

- ¿Cómo valoraría sus acciones y ayuda en este sentido?

Fecha de recepción: 30 de marzo de 2017.

Fecha de revisión: 25 de octubre de 2017.

Fecha de aceptación: 25 de octubre de 2017. 
